\title{
Research on Control Strategy for DFIG Connected to DC-Microgrids
}

\author{
Ji Peng ${ }^{1}$, Zhao Deren ${ }^{1}$, Xia Fei ${ }^{1}$, Xia Zongze ${ }^{1}$, Huang Xiaobo ${ }^{1}$, Cong Peixian ${ }^{1}$, Yang Zhixiong ${ }^{2}$, \\ Di Zhuo ${ }^{1}$, Song $\mathrm{Li}^{1}$ \\ ${ }^{1}$ State Grid Liaoyang Electric Power Supply Company, Liaoyang 111000, China) \\ ${ }^{2}$ Kunming Institute of Physics, Kunming 650223, China) \\ E-mail: xiongmaoer39@126.com
}

Keywords: DC-Microgrids; Torque ripple; Resonant controller; Torque ripple; Grid connected power; DFIG

Abstract: Aiming to the voltage of the stator side is generated by the modulation of the SSC in the improved topology, especially under the circumstance with the asymm Teric fault of stator side, DFIG's electromagn Teic torque, amplifies ripple of grid-connected power for the grid side. The novel control method suitable to stator side converter and rotor side converter based on reduced-order resonant controller (RORC) is proposed in this paper, DFIG's torque and output power performance are improved. Under the RORC control conditions the transfer functions of stator current and torque control system are established, the amplitude characteristic and the system stability of RORC control are analyzed. The simulation results in Matlab/Simulink verify the correctness and validity of the proposed method.

\section{Introduction}

Under the above conditions, the voltage, current, flux linkage, power and torque of the generator unit all contain the fluctuation component, which is not conducive to the stable operation of the unit and the power quality of the unit output. ${ }^{[1]}$ The research results show that the electromagnetic torque is one of the most important control parameters of the DFIG unit, and its fluctuation will increase the fatigue of the transmission shaft and reduce the operating performance and service life of the unit. In the new study, literature and propose a method to suppress the torque ripple resonant loop regulation based on, but this method has some limitations; this paper discusses the mechanism of the DFIG ${ }^{[2]}$ generation unit two harmonic torque torque fluctuation component, established the stator current and stator output power and torque of the qualitative relationship.

\section{DFIG Mathematical Model with Negative Sequence Component}

$$
\mathcal{U}_{s d q}^{+}=\mathcal{u}_{s d q+}^{+}+\mathcal{U}_{s d q-}^{+}=\mathcal{U}_{s d q+}^{+}+\mathcal{U}_{s d q_{-}}^{-} e^{-j 2 \mathcal{W}_{1} t}
$$




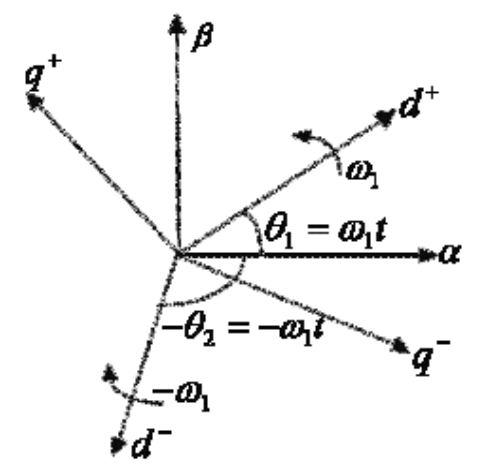

Fig.1 Positive and negative sequence component space vector

In the formula, the superscript + , - represents the positive and negative components in the synchronous velocity coordinate system. The corresponding DFIG stator current and stator flux can be written as:

$$
\begin{aligned}
& i_{s d q}^{+}=i_{s d q+}^{+}+i_{s d q-}^{+}=i_{s d q+}^{+}+i_{s d q-}^{-} e^{-j 2 \mathcal{W}_{1} t} \\
& \Psi_{s d q}^{+}=\Psi_{s d q+}^{+}+\Psi_{s d q-}^{+}=\Psi_{s d q+}^{+}+\Psi_{s d q-}^{ \pm} e^{-j 2 \mathcal{W}_{1} t}
\end{aligned}
$$

According to formula (2), (3), the Te expression of the electromagnetic power Pe and the electromagnetic torque:

$$
\begin{aligned}
& P_{e}=1.5 w_{r} \operatorname{Im}\left(\Psi_{s d q}^{+} i_{s d q}^{+}\right)=P_{e 0}+P_{e 2} \\
& T_{e}=-1.5 n_{p} \operatorname{Im}\left(\Psi_{s d q}^{+} i_{s d q}^{+}\right)=T_{e 0}+T_{e 2}
\end{aligned}
$$

In the formula: $\mathrm{Pe} 0$ and $\mathrm{Te} 0$ are respectively the constant component of the electromagnetic power $\mathrm{Pe}$ and the electromagnetic torque $\mathrm{Te} . \mathrm{Pe}_{2}$ and $\mathrm{Te}_{2}$ are respectively the $100 \mathrm{~Hz}$ fluctuation components of the electromagnetic power Pe and the electromagnetic torque Te.

If the Ps and Pr are the active power output of the generator set and the rotor side, and the iron loss and copper loss of the unit, the Pe expression of the electromagnetic power of the unit is:

$$
P_{e} \approx P_{s}+P_{r}=P_{s}-\mathrm{s} P_{s}
$$

The combined type (4), (5) can be obtained, the electromagnetic torque Te of the DFIG unit and the stator side active power Ps satisfy the relation

$$
T_{e}=\frac{n_{p}}{w_{1}}(1-s) P_{s}
$$

Combined with the formula (1), (2), the output power Ps of the stator side of the generator satisfies the relation

$$
\begin{aligned}
& P_{s}=\frac{1.5}{1-s} \operatorname{Im}\left(\Psi_{s d q}^{+} i_{s d q}^{+}\right)=P_{s 0}+P_{s 2} \\
& P_{s 0}=\frac{1.5}{1-s} \operatorname{Im}\left(\Psi_{s d q}^{+} i_{s d q}^{+}+\Psi_{s d q-}^{-} i_{s d q-}^{-}\right) \\
& P_{s 2}=\frac{1.5}{1-s} \operatorname{Im}\left(\Psi_{s d q+}^{+} i_{s d q-}^{-} e^{j 2 \mathcal{W}_{1} t}-\Psi_{s d q}^{-} i_{s d q+}^{+} e^{-j 2 \mathcal{W}_{1} t}\right)
\end{aligned}
$$

Type: $\mathrm{Ps}_{0}, \mathrm{P}_{\mathrm{s} 2}$ constant active power DFIG generator stator side output and two harmonic wave component; constant component similarly can write unit stator output reactive power Qs and two 
harmonic component expressions. If the formula (6) and (7) are known, the electromagnetic torque and power of the unit can be satisfied:

$$
\begin{aligned}
& T_{e 0}=\mathrm{T} P_{s 0} \\
& T_{e 2}=\mathrm{T} P_{s 2} \\
& T_{e}=\mathrm{T}\left(P_{s 0}+P_{s 2}\right)
\end{aligned}
$$

From Fig. 2, DFIG stator negative sequence voltage and current is mainly caused by the electromagnetic torque fluctuation component, while suppressing unit torque ripple components have smooth output to power, in order to improve the stability of the DFIG unit.

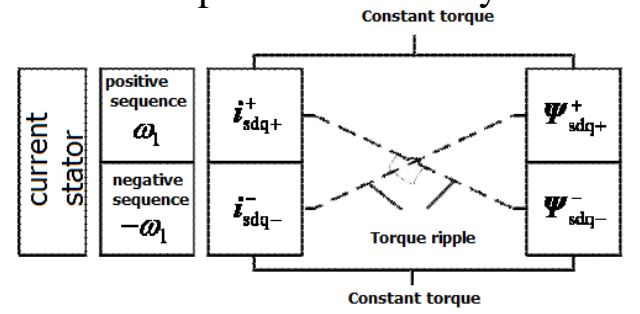

Fig. 2 The relationship between torque and stator current and flux

\section{Torque Ripple Suppression Strategy Implementation of DFIG Unit}

\subsection{Torque ripple suppression principle of DFIG unit}

In the improved RSC control strategy, the effect of RC on the stator current feedback loop, the two harmonic component of stator current suppression, by formula (7), (8), can indirectly inhibit the generator electromagnetic torque fluctuation of the two multiplier effect; in the improved SSC control strategy, RC for torque and power the feedback loop, by type (8), the two harmonic component of electromagnetic torque can directly inhibit the unit.

The resonant controller RC is the key step to ensure the torque ripple of the DFIG unit, which is shown in figure $3 .{ }^{[3]}$ Common resonant controller mainly reduced order and two order two, because the two order resonant controller will introduce stator current three harmonic component of large, more important is the order of the unit control system increases with the introduction of RORC, is not conducive to the stability of the control system, ${ }^{[4]}$ this paper uses a reduced order resonant controller RORC and its transfer function as shown in (9).

$$
\mathrm{G}(\mathrm{s})=k_{r} \frac{w_{c}}{s+\left(w_{c}+j 2 w_{1}\right)}
$$

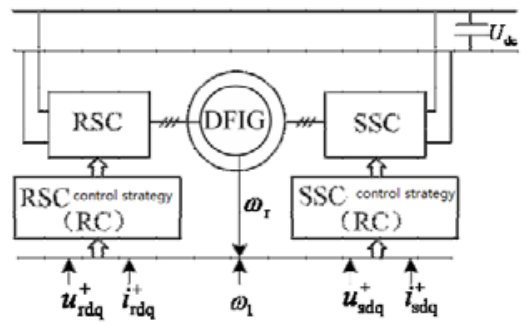

Fig. 3 schematic diagram of electromagnetic torque ripple suppression of DFIG unit

Formula: $k_{r}$ is the coefficient; $\omega_{1}$ is the reference angle frequency; $\omega_{c}$ is the cut-off angle frequency, the general value of $5 \sim 25 \mathrm{rad} / \mathrm{s}$. As for type (9) are shown in descending order resonant 
controller, with the increase of the coefficient rk at the resonant frequency, the bandwidth of about increased, can enhance the resonant controller of unit stator voltage frequency offset adaptability, no change in phase frequency characteristics; at the resonant frequency, two times frequency AC signal amplitude attenuation. ${ }^{[5]}$ The electromagnetic torque ripple suppression method of DFIG unit RORC, does not depend on the electrical parameters of the wind turbine, the universal method of application; at the same time without separation and extraction of the unit rotor side voltage and current of positive and negative sequence, to avoid a lot of complicated calculation and control delay.

\subsection{RSC control strategy based on RORC}

For RSC control, using the improved SFO control method, a rotor voltage equation and counter the stator excitation current for dynamic component

$$
\begin{aligned}
& \mathcal{U}_{r d}^{+}=R_{r} i_{r d}^{+}+L_{\sigma r} p i_{r d}^{+}-E_{r d} \\
& u_{r d}^{+}=R_{r} i_{r d}^{+}+L_{\sigma r} p i_{r d}^{+}+E_{r q}
\end{aligned}
$$

Type (10), $E_{r d}, E_{r q}$ is voltage decoupling, the expression for

$$
\begin{aligned}
& E_{r d}=w_{s} L_{\sigma r} i_{r d}^{+} \\
& E_{r q}=w_{s}\left(L_{m} \Psi_{s} / L_{s}+L_{\sigma r} i_{r d}^{+}\right)
\end{aligned}
$$

The expression for the type (10), (11):, $u_{r d}^{+}, u_{r q}^{+}$is respectively d, q axis components of the rotor voltage and rotor current respectively; $i_{r d}^{+}, i_{r q}^{+}$is the d, q axis component of the stator and rotor windings; $L_{m}$ is the equivalent mutual inductance between stator and rotor windings; Ls , Lr is the stator and rotor winding inductance, $\mathrm{Rr}$ is the rotor winding resistance; $\sigma$ is the leakage coefficient; $L_{\sigma r}=\sigma L_{r}, \omega_{s}$ is slip angular frequency; $\mathrm{P}$ is the differential operator. The introduction of resonant controller s G, combined with (10) (11), can be suppressed by the stator current of the second harmonic wave components of the RSC control strategy to achieve the block diagram, as shown in figure 4.

Figure 4: $i_{s a b c}^{+}$and $i_{\text {rabc }}^{+}$respectively for the ABC three-phase coordinate system of stator and rotor three-phase current; $i_{s d q}^{+}$and $i_{r d q}^{+}$respectively of the stator and rotor current DQ coordinates; $\omega_{r}$ is rotor frequency; $G_{R}(s)$ is introduced into the RSC control strategy of current feedback loop in RORC; the amount of superscript * corresponding variables the reference value.

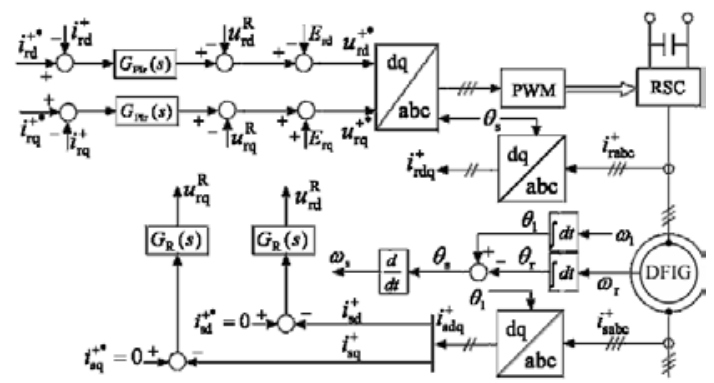

Figure 4 RSC control block diagram based on RORC

According to (9), (10) and figure 4, after the Laplace transform, simplify the DFIG unit stator 
current control equivalent block diagram, as shown in Figure 5. Figure 5 shows the following relations.

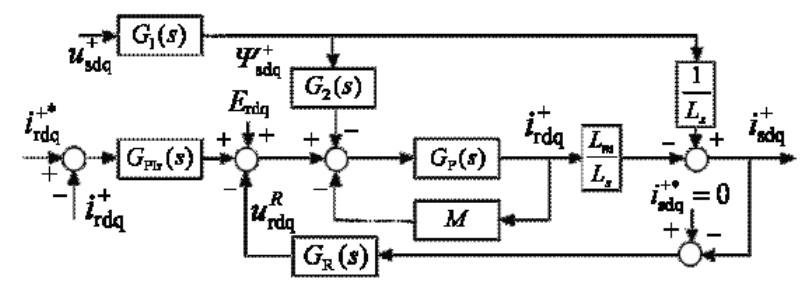

Fig. 5 equivalent diagram of stator current control of DFIG unit using RORC

\section{Operation Simulation}

According to figure.4 and figure 5 DFIG units RSC and SSC control block diagram, this paper builds a simulation model in MATLAB/Simulink, this chapter to verify the validity and effectiveness of the proposed control strategy, mainly discuss the inhibitory effect of control strategy of electrical power unit operation and electromagnetic torque fluctuation. The stator current d 、 q in Figure 5.4 is the same as the $G_{R}(s)$ parameter in the shaft feedback loop, $k_{r}$ is 100. The value of $\omega_{c}$ is 10 . The $G_{S}(s)$ parameter $k_{r}$ of the electromagnetic torque and reactive power feedback loop in Figure 5.7 is shown in figure 100. ${ }^{\omega_{c}}$ were taken 20 and 10 respectively.

\subsection{Operation simulation of DFIG}

In the simulation model built in the MATLAB/Simulink environment, if the electromagnetic torque ripple of the unit is not considered, the RORC link is suppressed, and the three-phase voltage of the stator side of the DFIG is us. Balanced conditions, simulation results are shown in Figure 6 shows that the generator stator three-phase voltage waveform is stable, the amplitude of $110 \mathrm{~V}$, frequency of $50 \mathrm{~Hz}$ sine wave; and A in the stator phase voltage curve of B compared to fig 6 stator voltage waveform of harmonic content increased, the reason lies in the fig 4. As shown in the RSC topology control, the electrical quantities were calculated by measuring the rotor current.

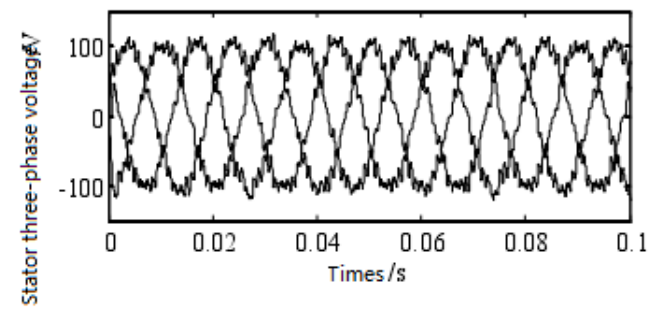

Fig. 6 Three-phase voltage waveform of stator side of DFIG unit

In order to verify the effectiveness of the control strategy of electromagnetic torque ripple suppression by RORC, the simulation results show that the three-phase voltage $U$. In the case of unbalance. In the simulation process, to simulate the DFIG stator side three-phase voltage U. In order to observe the effect of $\mathrm{A}$ on the electromagnetic torque ripple component of the unit, the phase voltage drop of the stator side of the A phase is taken as an example to observe the effect of RORC. The active power and reactive power are $1 \mathrm{KW}$ and $100 \mathrm{~V}\}$ A respectively.

In Figure 7, can be seen in the Os to 0.2S, US generator stator three-phase voltage balance, harmonic distortion is $11.26 \%$ THD, the two harmonic accounted for $20 \%$, Ps error range of [-3.16,4.70], QS wave interval error as the $\mathrm{Bu} 9.76,9.85]$; Te error range of 0 0.2S to 0.4s in 
0.33,0.21], A, 20\% phase voltage drop of stator three-phase voltage unbalance, harmonic distortion is $26.64 \%$ THD, the two harmonic content of about $40 \%$, increasing the power and torque fluctuation error, Ps error interval to bu 5.34,12.30]. Qs error interval to bu 17.29,16.30];
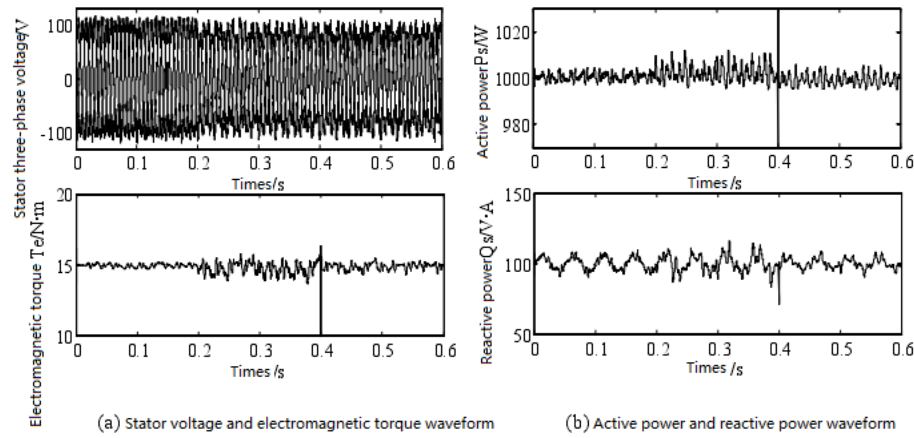

Fig. 7 Simulation results of DFIG unit under unbalanced voltage condition

Te error interval is $00.2 \mathrm{~S} \mathrm{Bu} \mathrm{1.34,0.78]} \mathrm{to} 0.4 \mathrm{~s}$, the control method proposed in this chapter, the RSC and SSC control strategy with RORC, from the simulation map It can be seen that the set of active and reactive power fluctuation error relative to $0.4 \mathrm{~s} 0.2 \mathrm{~S}$ decreased, Ps error interval is [-5.18,6.00], Q, error interval time-varying [-9.89,9.16]; Te error interval [0.73,0.51].

\subsection{Comparative analysis of simulation results}

As can be seen from table 5.4, the stator side of the three-phase voltage U. Unbalance, the negative sequence component of voltage and current causes the fluctuation error of output power and electromagnetic torque $\mathrm{Te}$ of the unit increases, the percentage of the fluctuation error is $\mathrm{u}$, and the balance is increased, among which $\mathrm{U}$. The unbalanced reactive power $\mathrm{Q}$ and electromagnetic torque of Te, a greater impact on the unit; for the control strategy and RORC, error and fluctuation of power and electromagnetic torque fluctuation percentage of Te decreased significantly.

Table 1 Statistical data of simulation results

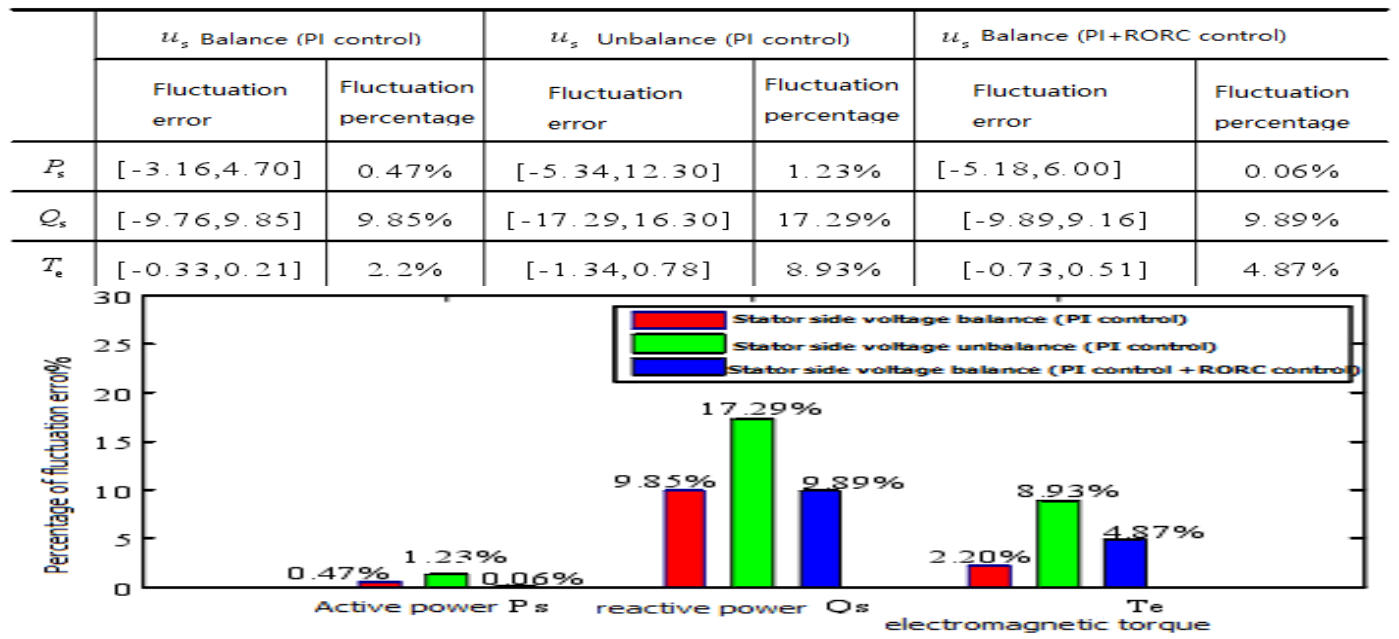

Fig. 8 comparison of PI and PI+RORC control unit operating error

It can be seen in Fig. 8, RORC RSC and SSC improved control strategy can effectively suppress the unit stator three-phase voltage unbalance torque ripple caused by the fluctuation based on the error percentage decreased from $8.93 \%$ to $4.87 \%$, reduce the unit shaft fatigue; at the same time on the DFIG machine stator output active and reactive power fluctuation to inhibit the effectiveness of 
the proposed control method is verified; at the same time as in Figure 7 in frequency characteristic curve, $100 \mathrm{~Hz}$ resonant frequency the amplitude gain is not strictly zero, so PI+RORC control strategy did not completely inhibit the electromagnetic torque fluctuation error.

\section{References}

[1] Dong Chen, Lie Xu, Liangzhong Yao. DC voltage variation based autonomous control of DC microgrids[J]. IEEE Transactions on Power Delivery, 2013, 28(2):637-648

[2] Changjin Liu, Frede Blaabjerg , Wenjie Chen Dehong Xu. Stator currentharmonic control with resonant controller for doubly fed induction generator[J]. IEEE Transaction on Power Electronics, 2012, 27(7): 3207-3220.

[3] Tine L. Vandoorn, bart meeraman, lieven degroote, et al. A control strategy for islanded microgrids with DC-link voltage control[J]. IEEE Transactions on Power Delivery, 2011, 26(2):703-713.

[4] Matteo F. Iacchetti, Gil D, Marques, Roberto. Torque ripple reduction in a DFIG-DC system by resonant current controllers [J]. IEEE Transactions on Power Electronics, 2015, 30(8): 4244-4254.

[5] G, D. Marques, M. F. Iacchetti, Air-gap power-based sensorless control in a DFIG connected to a DC-link[J]. IEEE Transactions on Energy Conversion, 2015, 30(1):362-375. 